\title{
Análise de corpora de Práticas Integrativas e Complementares em Saúde e proposta de um glossário bilíngue
}

\section{Analysis of corpora of Integrative and Complementary Practices in Health and proposal for a bilingual glossary}

Luana Mara Almeida Teixeira* Angela Maria Tenório Zucchi**

\footnotetext{
*Instituto Federal de Educação, Ciência e Tecnologia de São Paulo. E-mail: luana.mara@ifsp.edu.br ** Universidade de São Paulo. E-mail: angelazucchi@usp.br TradTerm, São Paulo, v.37, n. 1, janeiro/2021, p. 265-293 Número Especial - Linguística de Corpus www.revistas.usp.br/tradterm
} 
Resumo: 0 ser humano sempre buscou a recuperação e a manutenção da saúde. Além da medicina convencional ou alopática, existem numerosas formas complementares de tratamento, no Brasil chamadas Práticas Integrativas e Complementares em Saúde (PICS). Por meio da elaboração de corpora comparáveis em português e inglês, analisamos a linguagem utilizada por essa área de especialidade. Para realizar este trabalho, utilizamos como referenciais teóricos as bases da Linguística de Corpus tratadas por Berber Sardinha $(2004,2009)$ e Tagnin $(2013,2015)$ e fundamentos da Terminologia tratados por Barbosa (2004), Barros (2004), Bevilacqua (2005) e Krieger e Finatto (2004). Após abordarmos a compilação dos corpora, apresentamos alguns resultados e uma proposta de glossário bilíngue para os termos encontrados, com exemplos de verbetes. Esperamos contribuir com um material de apoio aos tradutores dessa área.

Palavras-chave: Linguística de corpus; Práticas Integrativas e Complementares em Saúde (PICS); Terminologia bilíngue; Tradução.

Abstract: The human being has always sought for recovery and maintenance of health. In addition to conventional or allopathic medicine, there are numerous complementary forms of treatment, known in Brazil as Integrative and Complementary Practices in Health. By creating comparable corpora in Portuguese and English, we analyze the language used in this specialized area. To this end, we use as main theoretical references Corpus Linguistics, according to Berber Sardinha (2004, 2009) and Tagnin (2013, 2015); and Terminology, according to Barbosa (2004), Barros (2004), Bevilacqua (2005) and Krieger and Finatto (2004). After explaining the compilation of the corpora, we present some results and a bilingual glossary proposal for the terms found, with examples of entries. We hope to contribute with material to support translators in this area.

Keywords: Corpus linguistics; Integrative and Complementary Practices in Health; Bilingual terminology; Translation.

TradTerm, São Paulo, v.37, n. 1, janeiro/2021, p. 265-293

Número Especial - Linguística de Corpus

www.revistas.usp.br/tradterm 


\section{Introdução}

Este artigo pretende descrever as etapas envolvidas na elaboração de um glossário bilíngue no par de línguas e variantes português brasileiro e inglês britânico, na direção português-inglês, da área de Práticas Integrativas e Complementares em Saúde (PICS). Para a realização deste trabalho, utilizamos como arcabouço teórico e metodológico a Linguística de Corpus (LC), que tem se mostrado muito pertinente para a elaboração de materiais de apoio aos tradutores, ao possibilitar a análise de grandes quantidades de textos de forma computadorizada e permitir acurada observação da língua em uso, como se pode verificar em Viana e Tagnin (2015). A utilização de corpora tem grande aplicação na confluência das áreas de Terminologia e Lexicografia para a compilação de produtos terminográficos, como glossários e dicionários no âmbito de diferentes línguas de especialidades.

Sobre o tema escolhido para a elaboração do glossário, são necessárias algumas observações. Em primeiro lugar, é preciso distinguir medicina alopática (vinculada à prescrição de medicamentos farmacêuticos) de Medicina Tradicional. Para a Organização Mundial da Saúde (OMS) ${ }^{1}$ (WHO 2002), Medicina Tradicional (MT) é um termo utilizado para se referir tanto a sistemas como a Medicina Tradicional Chinesa, a Ayurveda Indiana e a Unami Árabe, quanto às várias formas de medicina indígena. A MT inclui terapias com uso de medicação (envolvendo a ingestão de remédios à base de ervas, partes de animais ou minerais) e terapias sem medicação, como no caso da acupuntura, terapias manuais e terapias espirituais.

A Medicina Tradicional é frequentemente denominada Medicina Complementar, Alternativa ou Não Convencional em países onde o sistema de saúde é baseado na medicina alopática, ou onde a MT não foi incorporada ao sistema nacional de saúde.

No Brasil, essas técnicas terapêuticas foram chamadas de Práticas Integrativas e Complementares em Saúde (PICS), definidas pela Portaria de $\mathrm{n}^{\circ}$ 971, de 3 de maio de 2006 do Ministério da Saúde/Gabinete do Ministro, que

\footnotetext{
${ }^{1}$ OMS em português e WHO em inglês. Foi consultada a versão em inglês do documento, por isso a sigla WHO - World Health Organization, como consta nas referências.
}

TradTerm, São Paulo, v.37, n. 1, janeiro/2021, p. 265-293

Número Especial - Linguística de Corpus

www.revistas.usp.br/tradterm 
aprova a Política Nacional de Práticas Integrativas e Complementares (PNPIC) no Sistema Único Saúde (SUS).

Posteriormente, foi publicada a Portaria $\mathrm{n}^{\circ} 849$, de 27 de março de 2017 do Ministério da Saúde, que incluiu mais catorze técnicas terapêuticas na PNPIC, como, por exemplo, Arteterapia, Ayurveda, Meditação e Osteopatia, e em março de 2018 foram incluídas mais dez modalidades de PICS, totalizando 29 práticas reconhecidas no país.

Embora a PNPIC - SUS não englobe todas as variedades de terapias complementares, alternativas ou não convencionais, optamos por utilizar a nomenclatura Práticas Integrativas e Complementares em Saúde (PICS) neste trabalho para nos referirmos a todos esses tipos de práticas em saúde, buscando manter a terminologia que já foi institucionalizada por órgãos governamentais.

De acordo com a OMS (WHO 2013), a utilização de Medicina Complementar ou Alternativa tem sido cada vez mais objeto de estudos para sua regulamentação em diversos países, além de ter aumentado sua inserção em cursos de nível superior nas últimas décadas. No entanto, mesmo com o aumento de pesquisas na área e a necessidade de uma terminologia padronizada para tratar dessa temática, faltam materiais de apoio para tradutores, pesquisadores e profissionais do ramo, especialmente baseados na metodologia aqui descrita.

Dada essa lacuna, colocou-se o objetivo da análise de termos das Práticas Integrativas e Complementares em Saúde e a elaboração de uma proposta de glossário bilíngue com o par linguístico português brasileiro e inglês britânico, observando o uso dos termos em textos escritos originalmente em língua portuguesa para verificar a frequência e os contextos em que ocorrem, bem como a equivalência dos termos em inglês, também analisando textos nesse idioma. Este artigo traz parte dos resultados de pesquisa concluída em dissertação de mestrado, conforme Teixeira (2018).

Apresenta-se dividido em cinco partes. A primeira consiste nesta introdução com a justificativa do tema escolhido, a segunda expõe a fundamentação teórica envolvida na pesquisa, a terceira descreve a

TradTerm, São Paulo, v.37, n. 1, janeiro/2021, p. 265-293

Número Especial - Linguística de Corpus

www.revistas.usp.br/tradterm 
metodologia empregada na compilação dos corpora, a quarta apresenta a discussão dos resultados e a quinta traz um modelo de glossário com alguns exemplos. Por fim, abordamos as considerações finais.

\section{Terminologia e Linguística de Corpus para}

\section{Tradução}

Passamos a seguir à definição de alguns conceitos utilizados neste artigo, sendo o primeiro a Terminologia, que é o "conjunto de termos próprios de um domínio, de um grupo de pessoas ou de um indivíduo" (BoutinQUESNEL1985: 17) e tem como unidade-padrão o termo. Barbosa (2004: 58) ressalta que "termo" encerra o conjunto formado pela denominação e o conceito que esta denominação comporta para quem a interpreta. Podemos exemplificar com o termo meridiano, que possui conceitos diferentes para os profissionais no âmbito das PICS e para aqueles no domínio da Geografia.

Barros (2004: 40) define "termo" como uma "unidade lexical com um conteúdo específico dentro de um domínio especializado". Ao tratar dos tipos de obras lexicográficas e terminográficas, a autora distingue dicionário terminológico, no qual devem constar as definições, e glossário, que consiste em uma lista de unidades lexicais ou terminológicas acompanhadas de seus equivalentes em língua(s) estrangeira(s) e que tem como principal característica a ausência de definições (BARROs 2004: 144).

No glossário que vamos apresentar, optamos por manter essa característica de não acrescentar definições autorais, porém, selecionando exemplos de contexto de uso que forneçam ao leitor informações relevantes para a compreensão do termo e, sempre que estivessem disponíveis nos corpora, incluindo exemplos que apresentem definições.

Com relação à constituição lexical, os termos podem ser simples (ex. meridiano, aura) ou complexos (ex. terapias complementares, acupuntura sistêmica). De acordo com Krieger e Finatto (2004: 81), os termos complexos são também chamados de sintagmas terminológicos e abundam nas comunicações especializadas. Abordando a Terminologia do ponto de vista

TradTerm, São Paulo, v.37, n. 1, janeiro/2021, p. 265-293

Número Especial - Linguística de Corpus

www.revistas.usp.br/tradterm 
fraseológico, Bevilacqua (2005: 80) cita também as denominações "unidades sintagmáticas", "termos complexos ou sintagmáticos", e elege "unidade fraseológica especializada (UFE)" especialmente para abarcar os fraseologismos que podem ser mais extensos que duas unidades, incluindo verbo e advérbio, e que ocorrem necessariamente com um termo. Nesta pesquisa, optamos por usar "termos complexos".

Almeida (2006: 88) aponta etapas importantes a serem cumpridas para a construção de um produto terminológico (glossário, dicionário, mapa conceitual etc.). A autora reforça a necessidade do cumprimento de certos requisitos para garantir a confiabilidade e validade do corpus utilizado, reunindo as características levantadas por diversos pesquisadores em: "autenticidade, representatividade, balanceamento, amostragem, diversidade e tamanho".

Ainda em relação à geração de glossários especializados, Krieger e Finatto (2004) descrevem várias etapas fundamentais, como critérios de confiabilidade e organização, questões como o sistema de remissivas e de que forma serão apresentadas as equivalências em língua estrangeira, entre outras.

Dentre vários aspectos importantes na elaboração de um glossário, destacamos a microestrutura, referente às escolhas de inclusão e organização dos dados de cada entrada no repertório, e a macroestrutura, relacionada à concepção geral da obra e o conjunto das partes principais que a compõem, como introdução, anexos (quando houver), bibliografia, organização dos verbetes de forma semasiológica (ordem alfabética) ou onomasiológica (temática).

Uma parte fundamental da macroestrutura é a nomenclatura, conjunto de todas as entradas do glossário. Para sua composição, podem ser levados em consideração fatores qualitativos, como a pertinência do termo para a área de conhecimento estudada, e quantitativos, com base na frequência de uso do termo. Pode acontecer de alguns termos levantados estatisticamente não serem fundamentais à pesquisa, então devem-se aliar fatores qualitativos e quantitativos, ponderando sobre a real representatividade dos termos

TradTerm, São Paulo, v.37, n. 1, janeiro/2021, p. 265-293

Número Especial - Linguística de Corpus

www.revistas.usp.br/tradterm 
selecionados dentro de uma área de conhecimento. Para isso, é preciso aliar o embasamento teórico-metodológico da pesquisa com familiaridade profissional na linguagem de especialidade em questão.

Com uma perspectiva empírica para o estudo da língua em diversas dimensões, a Linguística de Corpus (LC) é especialmente útil no estudo da Tradução, por permitir o exame de numerosas ocorrências em língua natural e a observação de repetições e padrões lexicais ou gramaticais, com fundamentos estatísticos e recursos computacionais (TAGNIN 2015: 19).

Devido a essa vantagem de analisar a linguagem em seu uso autêntico, de forma ampla e sistematizada, consideramos a LC a metodologia mais adequada para o desenvolvimento deste trabalho. Atrelada ao desenvolvimento da tecnologia, permite a observação de uma grande quantidade de textos (corpus) em formato eletrônico, passível de análise por meio de ferramentas computacionais específicas. Assim, dados quantitativos são gerados, permitindo um aprofundamento da investigação além da intuição do pesquisador.

De acordo com Tagnin (2013: 216), corpus designa uma "coletânea de textos entendidos num sentido amplo, em formato eletrônico, compilados segundo critérios específicos para o estudo a que se propõem". Dentre os vários tipos de corpora existentes, compilamos para esta pesquisa corpora comparáveis bilíngues, que apresentam textos originais nos dois idiomas de estudo, ou seja, português brasileiro e inglês britânico.

Para a análise dos corpora, há recursos computacionais específicos. No Brasil, são três os mais conhecidos: o Antconc, software gratuito desenvolvido por Laurence Anthony, da Faculdade de Ciências e Engenharia da Universidade de Waseda; Sketch Engine, um gerenciador de corpora e software para análise, idealizado por Adam Kilgarrif e produzido por outros especialistas da Lexical Computing; e o WordSmith Tools ${ }^{2}$ (WST), criado por Mike Scott, da Universidade de Liverpool. Nossa escolha recaiu sobre esse último por ser amplamente utilizado nas universidades brasileiras e por não necessitar de assinatura mensal, mas sim uma única aquisição e instalação na máquina.

\footnotetext{
${ }^{2}$ https://lexically.net/wordsmith/version6/

TradTerm, São Paulo, v.37, n. 1, janeiro/2021, p. 265-293

Número Especial - Linguística de Corpus

www.revistas.usp.br/tradterm
} 
O WST possui três ferramentas básicas (BERBER SARDINHA 2009): WordList produz listas de palavras a partir do corpus compilado, ordenadas alfabeticamente ou por frequência; KeyWords extrai palavras que, mesmo com frequência relativamente baixa, são relevantes no corpus de estudo, em comparação a um corpus de referência; Concord realiza concordâncias, ou seja, uma listagem com segmentos de texto onde uma palavra selecionada ocorre.

Tendo em vista a compilação e análise dos corpora, são necessárias várias etapas. Primeiramente, é preciso fazer o planejamento do corpus, estabelecendo características necessárias para atender aos objetivos aos quais ele se destina. Mesmo assim, podem ser necessários ajustes ao longo da pesquisa, possibilitando que ocorra um balanceamento, que é "o processo pelo qual se garante que um corpus seja compilado de forma a manter um equilíbrio entre os tipos textuais que o compõem" (TAGNIN 2015: 321).

Após a coleta, dependendo da ferramenta de análise utilizada, há vários processos envolvidos na compilação do corpus, como os descritos por Magalhães (2014), que envolvem: conversão dos arquivos em formato .pdf para .txt com a função Optical Characters Recognition (OCR) e revisão sentença por sentença em toda a extensão do texto para verificar a ocorrência e corrigir possíveis erros de OCR. Essa etapa é necessária ao usar o Antconc e o WordSmith Tools, já o Sketch Engine faz análises a partir de corpus compilado com documentos em pdf.

Durante a etapa de seleção das palavras que podem ser candidatas a termo, é necessário utilizar um corpus de referência, geralmente de língua geral. Com as ferramentas computacionais, é feita uma comparação do conteúdo do corpus de estudo da língua de especialidade com o conteúdo do corpus de referência, que é bem maior. Esse procedimento permite comparar a ocorrência de uma unidade lexical que se caracteriza como termo no âmbito de uma área específica, ou seja, a maior frequência no corpus de estudo indica que é "palavra-chave positiva", conforme denominação usada por Tagnin (2015: 325). Como corpus de referência em português, utilizamos o Lácio Ref e, em inglês, o British National Corpus (BNC).

TradTerm, São Paulo, v.37, n. 1, janeiro/2021, p. 265-293

Número Especial - Linguística de Corpus

www.revistas.usp.br/tradterm 
Finalmente, a etapa de análise dos resultados ocorre por meio das concordâncias, que são listas de todas as ocorrências da palavra de busca em contexto. Esses contextos possibilitam a busca por equivalentes em língua inglesa. No escopo deste artigo utilizamos a noção de equivalência funcional, conforme Bevilacqua (2013), como a utilização na tradução de elementos linguísticos e culturais que expressem a mesma relação semântica e os mesmos efeitos na língua de partida e de chegada.

Para compor a nomenclatura - conjunto de todas as entradas - de um glossário, podem ser levados em consideração fatores como a pertinência temática e pragmática. Dada a relevância das denominações dos tipos de tratamentos relativos às PICS nas duas línguas escolhidas, nesta pesquisa, focamos o estudo do uso de substantivos e sintagmas nominais, inserindo no glossário diferentes modalidades de técnicas terapêuticas utilizadas.

\section{Metodologia e materiais utilizados}

Optamos pela utilização de textos de linguagem escrita para a construção de corpora comparáveis bilíngue no âmbito das Práticas Integrativas e Complementares (PICS). Para possibilitar a variabilidade de gêneros textuais, utilizamos quatro gêneros: artigos acadêmicos, livros, sites e legislação. A seguir, detalharemos como foram selecionados os textos em ambos os idiomas.

\subsection{Constituição do corpus em português}

Os artigos acadêmicos foram escolhidos por poderem demonstrar na prática a linguagem utilizada por pesquisadores, em sua maioria, advindos de áreas da saúde e afins. Os artigos também oferecem a vantagem de serem, geralmente, avaliados por seus pares, o que possibilita uma melhor precisão com relação aos termos empregados nessa área de especialidade.

TradTerm, São Paulo, v.37, n. 1, janeiro/2021, p. 265-293 Número Especial - Linguística de Corpus www.revistas.usp.br/tradterm 
Para realizar essa parte da pesquisa, utilizamos a Biblioteca Virtual da Saúde (BVS) ${ }^{3}$, disponível online, que realiza a busca em diversos repositórios de textos acadêmicos (artigos, monografias, teses etc.) catalogados como da área da saúde. 0 próprio site da BVS oferece a possibilidade de realizar alguns filtros nas buscas, como, por exemplo, idioma principal do artigo, ano de publicação, artigos completos disponíveis e país de afiliação.

Com a busca pelas palavras-chave "Terapias Complementares", acionamos os filtros para buscar apenas artigos em português, publicados dentro de um período de 20 anos (1995 a 2015), artigos completos disponíveis gratuitamente (aqueles que ofereciam gratuitamente apenas o resumo foram desconsiderados) e afiliados ao Brasil. Limitamos o número de artigos aos primeiros 100 , número que consideramos suficiente para a pesquisa. A bibliografia completa dos artigos utilizados está disponível no Apêndice em Teixeira (2018).

Nos casos em que o texto completo do artigo aparecia disponível no corpo do site, foi necessário apenas copiar e colar para Word e depois converter para o formato .txt. Nos casos em que o artigo estava disponível em .pdf, foi preciso extrair o texto por meio de OCR e/ou também copiar e colar, transformando o conteúdo em .txt.

Com relação aos livros utilizados, buscamos aqueles que foram publicados originalmente em português brasileiro (não utilizamos traduções) e verificamos se as publicações se caracterizavam como produção nessa variante da língua portuguesa.

Ao pesquisar pelas palavras-chave Medicina Integrativa, Terapias Alternativas e Complementares, Terapias Holísticas, Medicina Alternativa e Complementar, encontramos uma profusão de livros disponíveis em grandes livrarias do país. Para limitar a quantidade, decidimos selecionar os livros que se propunham a ser o mais abrangente possível, tratando das terapias e técnicas de forma geral e não abordando apenas uma ou algumas terapias específicas. Esse critério se coaduna com os objetivos de nossa pesquisa, ao

${ }^{3 \grave{A}}$ época da pesquisa,http://www.bireme.br/php/index.php. Atualmente direciona para http://bvsalud.org/.

TradTerm, São Paulo, v.37, n. 1, janeiro/2021, p. 265-293

Número Especial - Linguística de Corpus

www.revistas.usp.br/tradterm 
ensejar atingir o maior número possível de terapias utilizadas para a elaboração do glossário. Seguindo esses critérios, foram selecionados cinco livros, elencados nas referências de corpus em português.

Dentre esses livros, dois estavam em formato digital e três em formato impresso. Foi necessário escanear completamente os livros impressos e posteriormente utilizar o OCR para extração dos caracteres. Essa etapa foi bastante trabalhosa, pois, embora tenhamos contado com o apoio da tecnologia, foi preciso fazer também uma revisão manual, já que muitos caracteres foram reconhecidos erroneamente.

No que concerne aos textos de websites utilizados, mantivemos apenas os de instituições reconhecidas, pelo critério da confiabilidade, como hospitais e universidades que ofereciam atendimentos e cursos na área, na ocasião da pesquisa. A lista das instituições consta nas referências do corpus em português.

Em síntese, a quantidade de palavras de cada tipo de publicação ficou assim dividida:

Tabela 1: Constituição do corpus em português brasileiro

\begin{tabular}{|l|c|}
\hline \multicolumn{1}{|c|}{ Tipos de publicação } & Número de palavras \\
\hline Artigos acadêmicos & 396.313 \\
\hline Livros & 294.182 \\
\hline Legislação & 27.767 \\
\hline Sites & 11.100 \\
\hline Total & $\mathbf{7 0 4 . 1 7 9}$ \\
\hline
\end{tabular}

\subsection{Constituição do corpus em inglês}

Para manter os mesmos critérios de representatividade nos corpora, buscamos para a compilação do corpus em inglês britânico os mesmos tipos de publicações do corpus em português: artigos acadêmicos, livros, legislação (regulation) e textos de websites de instituições representativas. Procuramos manter o balanceamento por gêneros textuais e por tamanho de corpus. Dessa

TradTerm, São Paulo, v.37, n. 1, janeiro/2021, p. 265-293

Número Especial - Linguística de Corpus

www.revistas.usp.br/tradterm 
forma, ao realizar a busca por artigos acadêmicos, embora houvesse maior disponibilidade de artigos escritos em inglês do que em português, decidimos selecionar a mesma quantidade de artigos selecionados em português, ou seja, 100 artigos.

Em busca similar à realizada em português, para artigos em inglês do Reino Unido, utilizamos as palavras-chave Complementary Therapies e acionamos os filtros de busca por período (1995 a 2015), idioma do texto (inglês) e país de afiliação da revista ou periódico (Reino Unido). As referências completas dos artigos estão disponíveis no Apêndice em Teixeira (2018).

Quanto à publicação em livros, houve outras dificuldades além da grande quantidade disponível: não era possível identificar pela autoria se o texto pertencia à variante de inglês britânico; a aquisição demandava altos recursos financeiros; o tempo de entrega de importação era incompatível com o cronograma de realização da pesquisa. Por esses fatores, selecionamos apenas uma publicação, que consideramos bastante representativa, o manual Oxford Handbook of Complementary Medicine, com 424 páginas, escrito por pesquisadores e professores universitários da área de PICS de uma universidade britânica e publicado pela conceituada editora universitária britânica Oxford University Press

Com relação ao gênero Legislação/Regulation, utilizamos documentos oficiais do parlamento e de conselho regulador britânicos, como constam nas referências do corpus em inglês ao final.

No quesito textos de websites, além de material do site da associação britânica de terapeutas Federation of Holistic Therapists (FHT), selecionamos textos de hospitais e instituições de ensino que ofereciam tratamentos/cursos na área, como constam nas referências do corpus em inglês ao final.

Resumindo, a quantidade de palavras de cada modalidade textual ficou dividida da seguinte forma:

TradTerm, São Paulo, v.37, n. 1, janeiro/2021, p. 265-293 Número Especial - Linguística de Corpus www.revistas.usp.br/tradterm 
Tabela 2: Constituição do corpus em inglês britânico

\begin{tabular}{|l|c|}
\hline \multicolumn{1}{|c|}{ Tipos de publicação } & Número de palavras \\
\hline Artigos acadêmicos & 489.139 \\
\hline Livros & 80.575 \\
\hline Legislação & 124.767 \\
\hline Sites & 77.628 \\
\hline Total & $\mathbf{7 7 2 . 1 0 9}$ \\
\hline
\end{tabular}

\section{Análise dos resultados}

Após elaborarmos as listas de palavras nos dois idiomas e as compararmos com os corpora de referência para obter as listas de palavraschave, foi possível selecionar os termos referentes a tipos de terapias utilizados em PICS. Apresentamos nos gráficos a seguir as 25 primeiras modalidades de Práticas Integrativas e Complementares em Saúde nos corpora em português e em inglês, de acordo com a ordem de chavicidade, ou seja, o quanto esse termo é representativo para a área em questão. Em alguns casos percebemos que uma das PICS foi mais frequente no corpus (número de ocorrências maior), porém, sua chavicidade pode ter sido menor em comparação com o corpus de referência.

TradTerm, São Paulo, v.37, n. 1, janeiro/2021, p. 265-293

Número Especial - Linguística de Corpus

www.revistas.usp.br/tradterm 
Gráfico 1: As 25 primeiras PICS no corpus em português, por ordem de chavicidade

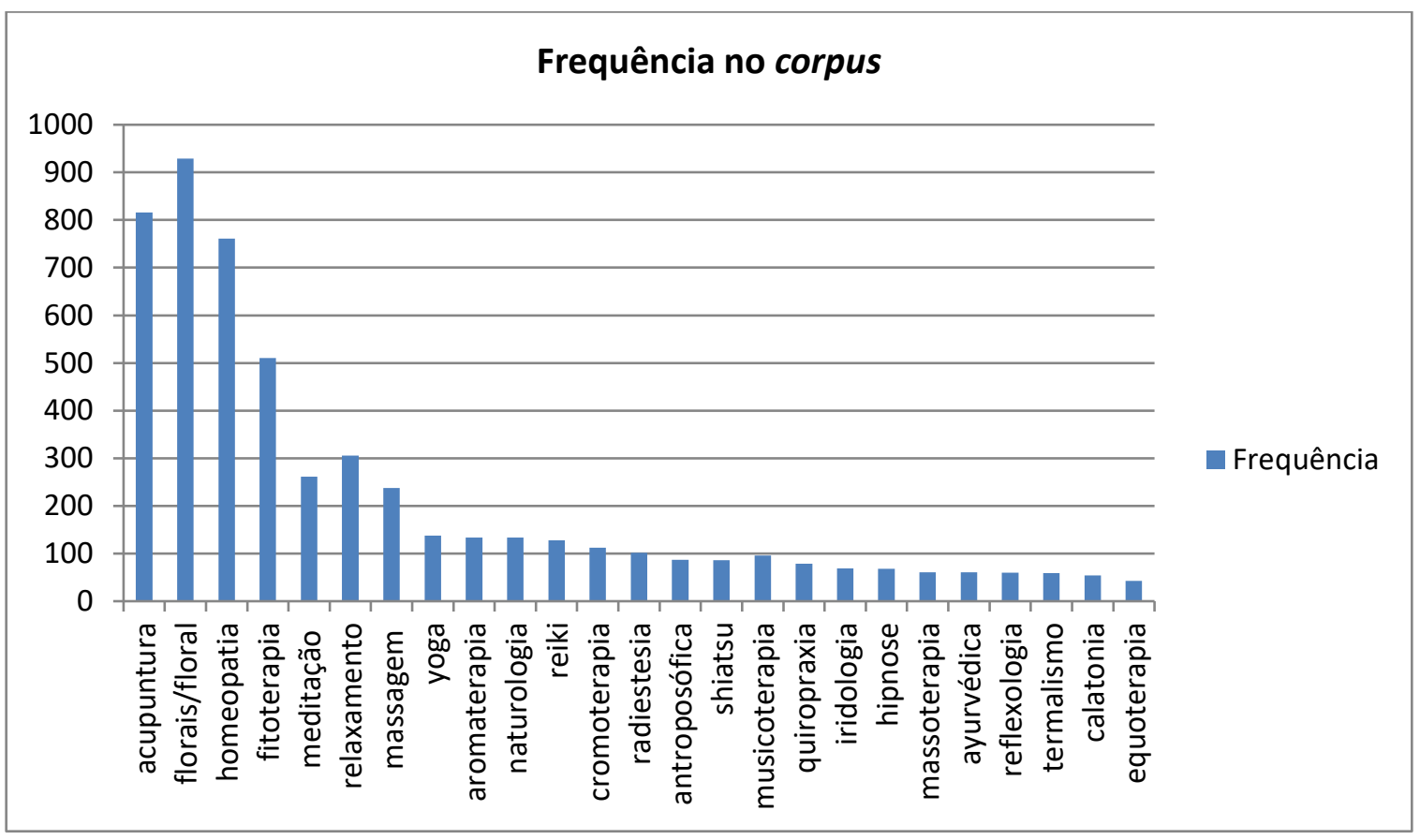

A ordem dos termos em português e em inglês em relação à chavicidade não coincidem, como se vê no gráfico a seguir:

Gráfico 2: As 25 primeiras PICS no corpus em inglês, por ordem de chavicidade

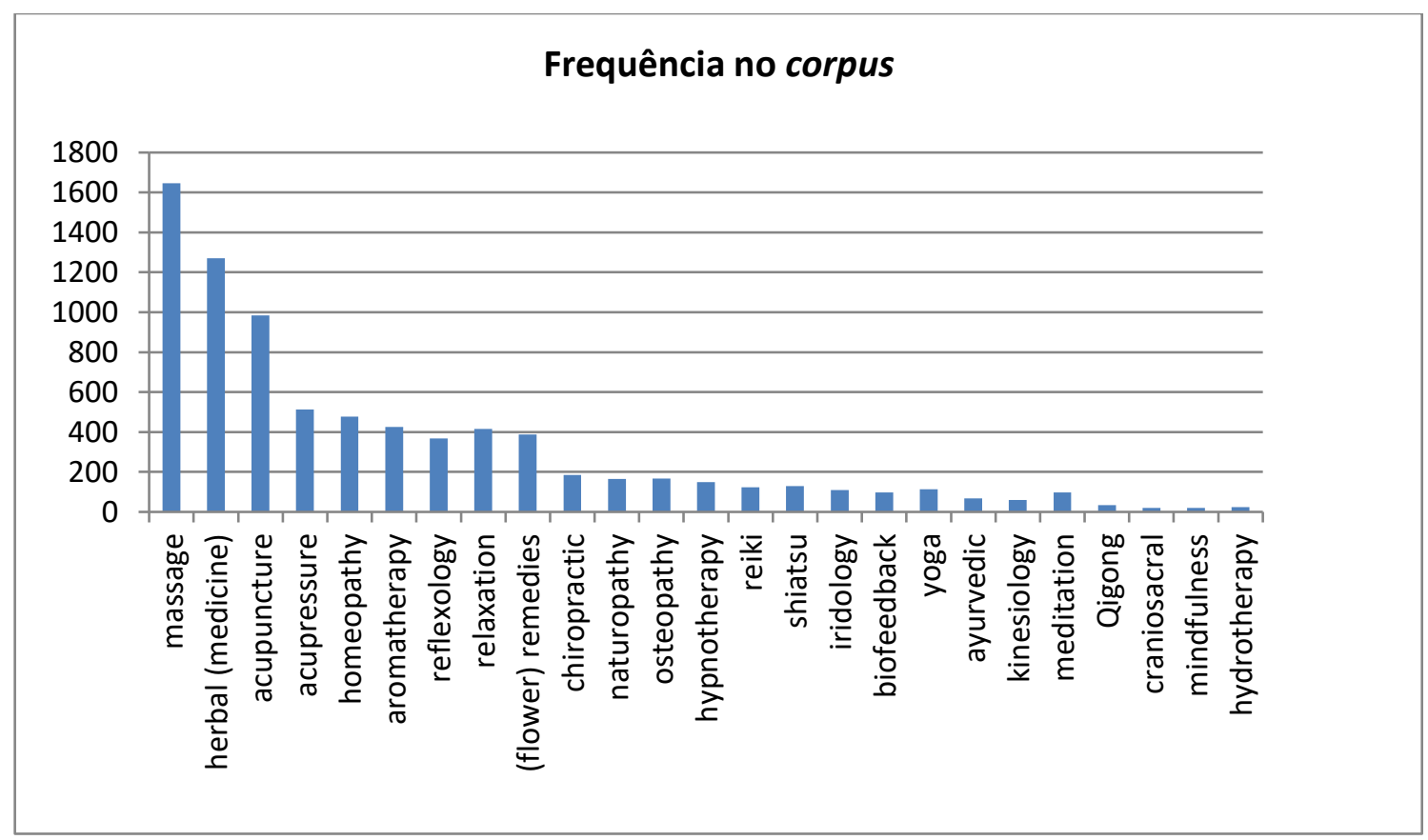

TradTerm, São Paulo, v.37, n. 1, janeiro/2021, p. 265-293

Número Especial - Linguística de Corpus

www.revistas.usp.br/tradterm 
Com a lista dos 25 primeiros termos referentes a PICS em português, buscamos os equivalentes em inglês. Realizamos o processo inverso com a lista em inglês. Alguns termos eram presentes em uma lista, porém, não na outra. Acupressure, por exemplo, constava entre as 25 primeiras PICS em inglês, enquanto seu equivalente em português, "acupressão", não estava entre essas primeiras, ainda que tenha ocorrido no corpus em português referindo-se ao mesmo tipo de terapia do termo em inglês. A partir dessas duas listas, unificamos os resultados, colocando os termos em ordem alfabética do português, alinhados com seus equivalentes em inglês, na seguinte tabela:

Tabela 3 - As primeiras PICS nos corpora em português e inglês

\begin{tabular}{|l|l|}
\hline acupuntura & acupuncture \\
\hline acupressão & acupressure \\
\hline (terapia) antroposófica & anthroposophyic therapy \\
\hline aromaterapia & aromatherapy \\
\hline (terapia) ayurvédica, Ayurveda & ayurvedic therapy, Ayurveda \\
\hline biofeedback & biofeedback \\
\hline calatonia & calatonia \\
\hline (terapia) craniossacral & kinesiology \\
\hline cromoterapia & craniosacral therapy \\
\hline equoterapia & colour therapy \\
\hline florais, (terapia) floral, floralterapia & equine therapy \\
\hline fitoterapia & $\begin{array}{l}\text { herber remedies, flower essences } \\
\text { phythotherapy }\end{array}$ \\
\hline iridologia & iridology \\
\hline hipnose, hipnoterapia & hypnotherapy, hypnosis \\
\hline homeopatia, (terapia) homeopática & homeopathy \\
\hline hidroterapia & hydrotherapy \\
\hline Massagem & massage \\
\hline massoterapia & massagetherapy \\
\hline meditação & meditation \\
\hline mindfulness & mindfulness \\
\hline musicoterapia & musictherapy \\
\hline
\end{tabular}

TradTerm, São Paulo, v.37, n. 1, janeiro/2021, p. 265-293 Número Especial - Linguística de Corpus www.revistas.usp.br/tradterm 


\begin{tabular}{|l|l|} 
naturologia & naturopathy \\
\hline osteopatia & osteopathy \\
\hline Qigong & Qigong \\
\hline quiropraxia & quiropraxy \\
\hline radiestesia & dowsing \\
\hline reflexologia & reflexology \\
\hline reiki & reiki \\
\hline relaxamento & relaxation \\
\hline shiatsu & shiatsu \\
\hline termalismo & balneotherapy \\
\hline yoga, ioga & yoga \\
\hline
\end{tabular}

Ao buscarmos os termos equivalentes em ambos os idiomas, percebemos a prevalência da utilização do mesmo radical, o que costuma ser bastante comum em linguagens técnicas e científicas. Quando não foi encontrado o equivalente prima facie, buscamos no próprio corpus os traços conceptuais da terapia que pudessem identificar o correspondente entre as duas línguas. Entendemos como traço conceptual características inerentes à modalidade terapêutica, sua forma de aplicação, materiais utilizados, local de origem ou criação. Exemplificamos, no quadro abaixo, os termos crenoterapia/termalismo e balneotherapy, em que o traço conceptual é o uso de águas minerais:

crenoterapia: (ver) termalismo

A Crenoterapia consiste na indicação e uso de águas minerais com finalidade terapêutica atuando de maneira complementar aos demais tratamentos de saúde (Brasil: Ministério da Saúde, 2006).

\section{balneotherapy}

In contrast to hydrotherapy, which generally employs normal tap water, balneotherapy is defined as baths using thermal mineral waters of at least $20^{\circ} \mathrm{C}$ temperature and a mineral content of at least $1 \mathrm{~g} / \mathrm{L}$ water from natural springs (Boddy, K. et al, 2) 
Já o termo 'radiestesia' possui um correspondente cognato radiesthesia, porém, o termo mais frequente em inglês para denominar esse tratamento é dowsing, e foi através da presença das palavras 'pêndulo' e 'pendulum', que é o instrumento utilizado para essa terapia, que chegamos a dowsing como equivalente para radiestesia.

O corpus foi compilado de forma balanceada e representativa, contudo, em alguns casos, mesmo seguindo o procedimento de busca de equivalentes acima mencionado, não foi possível encontrar um que fosse satisfatório, levando-nos a realizar pesquisas na Web para identificar a equivalência e buscar exemplos de uso, tendo em vista a elaboração do glossário bilíngue. Possivelmente, a ausência de alguns termos que denominam certas terapias pode estar relacionada a fatores culturais, ou seja, a terapia pode não ser amplamente adotada naquela cultura.

Observamos, tanto no corpus em português como no corpus em inglês, a tendência à utilização de nomes estrangeiros nas terapias originárias de culturas orientais: "reiki", do Japão; "shiatsu", da China; "Ayurveda" e "Yoga", da Índia. Em um primeiro momento, podem ter sido chamados "estrangeirismos", denominação usada para elementos de outro idioma, externos ao vernáculo e que, usados em contextos relativos a outra cultura, "imprimem uma cor local do país a que faz referência", conforme explica Alves (1990: 73) sobre neologismo.

Uma vez incorporados no acervo lexical, indicando denominações para práticas de uso já consolidadas no Brasil e no Reino Unido, são empréstimos com adaptação ortográfica ocidental, como demonstraram os corpora em inglês e português, sendo que em português ocorre também a variante "ioga", que se adequa às letras do alfabeto mais utilizadas em português.

Alguns pontos devem ser destacados em relação ao termo "fitoterapia". No corpus em português ele ocorreu 510 vezes, porém, o possível equivalente de mesmo radical, phytotherapy, ocorreu somente 15 vezes no corpus em inglês.

TradTerm, São Paulo, v.37, n. 1, janeiro/2021, p. 265-293 Número Especial - Linguística de Corpus www.revistas.usp.br/tradterm 
Verificamos, então, por meio das linhas de concordância, grande ocorrência de herbal medicine (315 vezes). Ao efetuar a busca por herbal medicine*, que possibilita o retorno da forma no plural, herbal medicines, as ocorrências totalizaram 567, enquanto herbalism ocorreu 44 vezes.

Há indícios no corpus de que herbal medicine seja o equivalente mais adequado para "fitoterapia", seguido de herbalism, enquanto phytotherapy, no corpus, é uma variante utilizada em menor escala. Dessa forma, podemos endossar o que diz Tagnin (2013: 19) sobre convencionalidade, como aquilo "que é aceito de comum acordo". Em inglês britânico vemos a preferência pelo uso de herbal medicine enquanto em português brasileiro se usa 'fitoterapia'. Seria possível uma tradução como phytotherapy, mas não é a mais frequente ou mais convencional no corpus analisado.

Em relação ao termo "florais", no plural, encontramos 674 ocorrências, enquanto a forma no singular, "floral", ocorreu 255 vezes. Dessas, 120 ocorrências foram do sintagma terminológico "terapia floral". A variante "floralterapia" apareceu 9 vezes no corpus. Em inglês apareceram as variações flower essence(s), 68 vezes, e (Bach) flower remedies, 36 vezes. É importante ter em mente que os florais de Bach são apenas um dos tipos existentes e disponíveis como terapia. Durante a etapa de compilação dos corpora, encontramos menção a mais de oitenta sistemas florais diferentes, desenvolvidos no Brasil e em diversos outros países.

\subsection{O termo terapia}

Ao analisarmos a lista de palavras-chave em português, observamos que o termo simples "terapias" apareceu na quarta posição, com 855 ocorrências. Sua forma no singular, "terapia", ocorreu 811 vezes, ocupando a décimasétima posição na lista de palavras-chave.

Ao aprofundarmos o estudo do referido termo, verificamos que ele dá origem a numerosos outros termos, formando modalidades de terapia por composição e, também, termos complexos, com mais de uma palavra. Excluindo os termos que não são específicos das PICS (como fisioterapia, psicoterapia, quimioterapia e radioterapia), encontramos 63 termos formados

TradTerm, São Paulo, v.37, n. 1, janeiro/2021, p. 265-293

Número Especial - Linguística de Corpus

www.revistas.usp.br/tradterm 
pelos processos de composição por aglutinação ou justaposição, como aromaterapia, arteterapia, cristalterapia, cromoterapia, dançaterapia, equoterapia, fitoterapia, floralterapia, hidroterapia, hipnoterapia, magnetoterapia, massoterapia, musicoterapia e reflexoterapia.

Com relação aos termos complexos, verificamos a prevalência de hiperônimos (termos que englobam várias modalidades terapêuticas): terapia alternativa, terapia complementar, terapia complementar e alternativa, terapia energética, terapia holística, terapia integrativa, terapia natural, terapia não convencional e terapia tradicional. Ocorreram ainda, em menor frequência, alguns termos complexos referentes a terapias específicas, como, por exemplo, terapia antroposófica, terapia floral e terapia ortomolecular.

Em busca similar no corpus em inglês, encontramos 5349 entradas para therap*, com ocorrências na forma singular (therapy) e plural (therapies), além de therapist e therapeutic.

Também realizamos a busca para *therapy (posição do asterisco que possibilita o retorno de termos compostos) e obtivemos o resultado de 2936 entradas. Excluindo-se aqueles que não são exclusivos da linguagem das PICS, como chemotherapy, physiotherapy, psychotherapy, radiotherapy, encontramos apenas sete termos referentes a modalidades terapêuticas específicas: aromatherapy, auriculotherapy, balneotherapy, hirudotherapy, hydrotherapy, hypnotherapy e phytotherapy.

Em compensação, os termos complexos foram abundantes em inglês, como se verificou também em português. Houve ocorrência de termos complexos na condição de hiperônimos, como alternative therapy, mind-body therapy, CAM therapy (sigla para complementary and alternative therapy), complementary therapy, energy therapy, holistic therapy, manipulative therapy, manual therapy, natural therapy e unconventional therapy. Como designação específica para as modalidades de PICS, encontramos 34 termos, por exemplo, anthroposophic therapy, art therapy, colour therapy, crystal therapy, equine therapy, flower essence therapy, herbal therapy, magnet therapy, massage therapy e music therapy.

TradTerm, São Paulo, v.37, n. 1, janeiro/2021, p. 265-293 Número Especial - Linguística de Corpus www.revistas.usp.br/tradterm 


\section{Proposta de glossário bilíngue}

Com os resultados obtidos, utilizamos alguns termos referentes a tipos de terapias e suas variantes para a demonstração de entradas no glossário. A macroestrutura adotada apresenta a lista de entradas de forma semasiológica, ou seja, em ordem alfabética.

A microestrutura do glossário apresenta o termo-entrada em português em negrito, possíveis variantes (outras denominações em português, se houver) por ordem de frequência no corpus, exemplos do termo inserido ao contexto de uso do corpus de língua original em português, fonte do contexto em português, contexto e fonte das variantes em português, se necessário.

Em seguida, aparece o termo correspondente em inglês, também em negrito, variantes em inglês (se houver) por ordem de frequência, exemplo de contexto de uso em inglês, fonte do contexto em inglês, exemplos das variantes e fonte do contexto em inglês, quando necessário, para facilitar a compreensão sobre a utilização do termo.

\subsection{Exemplos de verbetes do glossário bilíngue de Práticas Integrativas e Complementares em Saúde}

A seguir, exemplificamos o modelo de glossário, apresentando os verbetes em ordem alfabética, e seus equivalentes em inglês. Os termos são aqueles referentes às dez primeiras modalidades de PICS entre os 25 apresentados no gráfico do item 4. Salientamos que os exemplos não são tradução um do outro, mas sim contextos autênticos extraídos dos corpora de cada idioma.

\section{acupuntura}

A acupuntura emprega uma faceta da medicina tradicional chinesa e um de seus pilares é a teoria do Yin/Yang, outro é a teoria dos canais e colaterais (meridianos), que em número de 14 estão de tal forma envolvidos, que

TradTerm, São Paulo, v.37, n. 1, janeiro/2021, p. 265-293

Número Especial - Linguística de Corpus

www.revistas.usp.br/tradterm 
constituem uma verdadeira "malha energética" espalhada pelo corpo (TASHIROET AL, 2001).

acupuncture

Acupuncture aims to restore the balance of qi in the body, by inserting fine needles to stimulate specific 'acupoints' that run along each of the meridians (FHT, 2017).

\section{aromaterapia}

Aromaterapia é a arte e a ciência que visa promover a saúde e o bem-estar do corpo, da mente e das emoções, através do uso terapêutico do aroma natural das plantas por meio de seus óleos essenciais (BRITO, 2013).

aromatherapy

Aromatherapy is the systematic use of essential oils and absolutes in holistic treatments to improve physical and emotional well-being (GRCCT, 2017).

\section{fitoterapia}

Etimologicamente, vem das palavras gregas phyton (plantas) e therapeia (tratamento), ou seja, tratamento por meio das plantas (2). A fitoterapia caracteriza-se pela prática do uso de plantas, ou de suas partes, com a finalidade de prevenir, aliviar ou curar um processo patológico (BETTEGA, 2011).

herbal medicine, herbalism, phytotherapy

Herbal Medicine is the study of the medicinal qualities of plants, and their practical applications, to heal the symptoms and cause of disease ( $\mathrm{SCHOOL} \mathrm{OF}$ NATURAL MEDICINE UK, 2017).

Herbalism - Definition: The medicinal use of preparations that contain exclusively plant material (BODDY, K. ET AL, 2008).

florais, terapia floral, floralterapia

A partir dos ensinamentos de Edward Bach vários novos sistemas florais foram desenvolvidos em diversas partes do mundo, preparados com flores diferentes e em ecossistemas com propriedades peculiares e diferentes (COSTEIRA, 2015).

A Terapia Floral utiliza essências de flores, em extratos líquidos naturais e altamente diluídos (COSTEIRA, 2015).

TradTerm, São Paulo, v.37, n. 1, janeiro/2021, p. 265-293

Número Especial - Linguística de Corpus

www.revistas.usp.br/tradterm 


\begin{abstract}
flower essences, flower remedies
Flower essences are diluted extracts of various types of flowers and plants, similar in many ways to homeopathic remedies (FHT, 2017).

Bach and other flower remedies -- The theory behind flower remedies is that flowers contain the life force of the plant and this is imprinted into water through sun infusion which is used to make the flower remedy (HOUSE OF LORDS, 2017).
\end{abstract}

homeopatia; (medicina) homeopática

A homeopatia, sistema médico complexo de caráter holístico, baseada no princípio vitalista e no uso da lei dos semelhantes, foi enunciada por Hipócrates no século IV a.C. Foi desenvolvida por Samuel Hahnemann no século XVIII (BRASIL: MINISTÉRIO DA SAÚDE, 2006).

homeopathy; homeopathic medicine

Therefore, homeopathy is based on the idea of treating 'like with like' by administering hugely diluted versions of basically dangerous substances, such that a dose given to a patient may not contain even a single molecule of the active principle (HOUSE OF LORDS, 2017).

massagem

Ninguém duvida do poder relaxante de uma boa massagem, mas os profissionais da área ainda lutam para diferenciar uma simples sessão antiestresse (feita até no shopping) de uma intervenção capaz de fazer o corpo reagir a algum desconforto ou problema de saúde (ALMEIDA ET AL, 2015).

massage

Massage involves working the soft tissue of the body, to ease day-to-day stresses and muscular tension, and promotes relaxation (FHT, 2017).

meditação

Dentre essas técnicas, uma das mais conhecidas e estudadas é a meditação. 0 termo refere-se a uma variedade de práticas que visam focar ou controlar a atenção. Podem ser praticadas por muitos motivos, como promover calma e relaxamento físico, melhorar o equilíbrio psicológico, ajudar a enfrentar uma doença, ou estimular a saúde e o bem-estar geral (HOSPITAL SíRIO-LIBANÊS, 2017).

meditation

TradTerm, São Paulo, v.37, n. 1, janeiro/2021, p. 265-293

Número Especial - Linguística de Corpus

www.revistas.usp.br/tradterm 
Meditation: a series of techniques used to relax a patient to facilitate deep reflection and a clearing of the mind (HOUSE OF LORDS, 2017).

\section{naturologia}

A Naturologia estuda práticas e métodos naturais, tradicionais e modernos para o cuidado humano. Visa à expansão, manutenção e recuperação da saúde, bem como à melhoria da qualidade de vida e ao equilíbrio do ser humano com o ambiente e com a sociedade. Utiliza-se de práticas integrativas e complementares em saúde (UNIVERSIDADE DO SUL DE SC, 2017).

\section{naturopathy}

Naturopathy is a discipline of accumulated knowledge of effective natural therapies, diet and lifestyle in practice, and does not have a single originator (SCHOOL OF NATURAL MEDICINE UK, 2017).

\section{relaxamento}

Relaxamento - Técnica corporal desenvolvida com a finalidade de acalmar o corpo e a mente quando impregnados de estresse, tensão muscular e agitação mental, incompatíveis com o bem-estar e a paz necessários à vida dos seres humanos (COSTEIRA, 2015).

\section{relaxation}

Relaxation therapy - Definition: Techniques for eliciting the 'relaxation response' of the autonomic nervous system (BODDY, K. ET AL, 2008).

yoga, ioga

loga é um sistema filosófico-prático que conta com metodologias para a unificação dos diferentes elementos do psiquismo humano. Em todas as técnicas deste sistema o praticante busca eliminar sua agitação e ter um domínio harmônico de si mesmo (SILVA; LAGE, 2006).

yoga

Yoga is a series of poses and breathing exercises to work the body and relax the mind (FHT, 2017).

\section{Considerações finais}

Este artigo teve como objetivo apresentar uma análise da terminologia de Práticas Integrativas e Complementares em Saúde, com o uso de corpora, e

TradTerm, São Paulo, v.37, n. 1, janeiro/2021, p. 265-293

Número Especial - Linguística de Corpus

www.revistas.usp.br/tradterm 
descrever as etapas de elaboração de um modelo de glossário, buscando trazer uma contribuição à tradução dessa área de especialidade.

Concluímos que a metodologia utilizada ajudou a produzir um material de consulta mais condizente com o padrão de uso na área, como descrito em relação ao termo "fitoterapia", cujo equivalente mais frequente foi herbal medicine, demonstrando que a equivalência prima facie nem sempre está de acordo com o que é usado na prática.

Esperamos que ao realizar este estudo tenhamos colaborado com o desenvolvimento de materiais de apoio para a busca e utilização de termos adequados em português e inglês na área de conhecimento das Práticas Integrativas e Complementares em Saúde, para auxiliar o trabalho de tradutores, pesquisadores e terapeutas, que procuram promover a saúde e o bem-estar, algo tão necessário para o desenvolvimento de nossas potencialidades.

TradTerm, São Paulo, v.37, n. 1, janeiro/2021, p. 265-293

Número Especial - Linguística de Corpus

www.revistas.usp.br/tradterm 


\section{Referências}

Almeida, G. M. B. A Teoria Comunicativa da Terminologia e a sua prática. ALFA, N.50, V.2, São Paulo, 2006, pp.85-101.

Alves, I. M. Neologismo. Criação Lexical. São Paulo: Ática, 1990.

BARBOSA, M. A. Estrutura e formação do conceito nas línguas especializadas: tratamento terminológico e lexicológico. Revista Brasileira de Linguística Aplicada, v.4, n.1, 2004.

Barros, L. A. Curso Básico de Terminologia. São Paulo: EDUSP, 2004.

Berber Sardinha, T. Pesquisa em Linguística de Corpus com WordSmith Tools. Campinas: Mercado das Letras, 2009.

Berber Sardinha, T. Linguística de Corpus. São Paulo: Manole, 2004.

Bevilacqua, C. R.; TAgnin, S. E. O. (Orgs.). Corpora na Terminologia. São Paulo: HUB Editorial, 2013.

Bevilacqua, C. R. Fraseologia: perspectiva da língua comum e da língua especializada. Revista Língua e Literatura Frederico Westphalen, V.1011, 2005, pp. 73-86.

Bevilacqua, C. Por que e para que a linguística de corpus na terminologia. In: TAGNIN, S. E. O.; BEVILACQUA, C. (Org.) Corpora na terminologia. São Paulo: Hub Editorial, 2013, pp.11-45.

BOUTIN-QUESNEL, R. et al. Vocabulaire Systématique de la Terminologie. Québec: Publications du Québec, 1985.

BRITSH NATIONAL CORPUS. Disponível em: <http://corpus.byu.edu/bnc/>. Acesso em: 20 jun. 2015.

Krieger, M. G.; Finatto, M. J. B. Introdução à Terminologia: teoria de prática. São Paulo: Contexto, 2004.

MAGALHÃES, C. Estra: um corpus para o estudo do estilo da tradução. 2014. Disponível em:

https: / /periodicos.ufsc.br/index.php/traducao/article/view/2175-

7968.2014v2n34p248. Acesso em: 13 jul. 2015.

TAGNin, S. E. O. A Linguística de Corpus na e para a tradução. In: Viana, V.; TAGnIN, S. E. O. (Orgs.) Corpora na Tradução. São Paulo: Hub Editorial, 2015, pp.19-56.

TradTerm, São Paulo, v.37, n. 1, janeiro/2021, p. 265-293

Número Especial - Linguística de Corpus

www.revistas.usp.br/tradterm 
TAGnIN, S. E. O. Glossário de Linguística de Corpus. In: TAGNIN, S. E. O.; BeVILACQUA, C. (org.) Corpora na Terminologia. São Paulo: Hub Editorial, 2013.

TAGNIN, S. E. O. O jeito que a gente diz. Barueri: DISAL, 2013.

TEIXEIRA, L. M. A. Práticas integrativas e complementares: análise de corpora e glossário bilíngue português/inglês para tradutores. Dissertação de Mestrado, DLM, FFLCH. Universidade de São Paulo, São Paulo, 2018.

Viana, V.; TAGnin, S. E. O. (Orgs.) Corpora na Tradução. São Paulo: HUB Editorial, 2015.

World Health Organization. WHO traditional medicine strategy 2002-2005. Geneva: World Health Organization, 2002. Disponível em: <http://www.wpro.who.int/health_technology/book_who_traditional_m edicine_strategy_2002_2005.pdf>. Acesso em: 10 mai. 2015.

World Health ORganization. WHO traditional medicine strategy 2014-2023. Geneva: World Health Organization, 2013. Disponível em: <http://apps.who.int/iris/bitstream/10665/92455/1/9789241506090_en g.pdf?ua=1 >. Acesso em: 10 ago. 2016.

\section{Referências do corpus em português}

AlmeIDA, N. M. et al. (Org.). Manual de medicina integrativa: memento fitoterápico. Porto Alegre/RS: Simplíssimo Livros Ltda. 2015. 1ª Edição revista e ampliada.

COSTEIRA, O. Terapias naturais e holísticas: quais são, como foram criadas, para que servem, como são usadas. Curitiba/PR: Appris, 2015. $1^{\text {a }}$ Edição. 423 p.

KLEIN, M.; RODRIGUES, R. B. Guia completo das terapias alternativas: métodos terapêuticos naturais que proporcionam saúde integral. Joinville/SC:

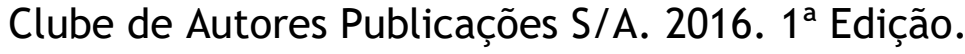

LIMA, P. T. Medicina integrativa: a cura pelo equilíbrio. São Paulo/SP: MG Editores, 2009. $2^{\mathrm{a}}$ Edição.

NAIFF, N. Curso completo de terapia holística \& complementar. Rio de Janeiro/RJ: Bestseller, 2016. $4^{a}$ Edição.

TradTerm, São Paulo, v.37, n. 1, janeiro/2021, p. 265-293

Número Especial - Linguística de Corpus

www.revistas.usp.br/tradterm 
Complexo Educacional FMU | FIAM-FAAM. Curso - Musicoterapia. Disponível em: <http://portal.fmu.br/curso/35/0/musicoterapia.aspx>. Acesso em: 17 abr. 2017.

SECRETARIA DE SAÚDE DO ESTADO DE GoIÁs. HMA muda de nome e amplia acesso às terapias alternativas. Goiânia, 21 mar. 2016. Disponível em: <http://www.saude.go.gov.br/view/4238/hma-muda-de-nome-eamplia-acesso-as-terapias-alternativas>. Acesso em: 12 abr. 2017.

Hospital Divina ProvidÊnCIA. Institucional - Responsabilidade social. Disponível em:<http://www.divinaprovidencia.org.br/institucional/index.php?id $=219$ \&idcategoria=10>. Acesso em: 12 abr. 2017.

Hospital IsRaeltta Albert Einstein. Pós-Graduação em Bases da Medicina Integrativa. Disponível em:

<https://www.einstein.br/ensino/pos_graduacao/bases_da_medicina _integrativa_sp>. Acesso em: 17 abr. 2017.

Hospital São Paulo (Unifesp). Grupo de Trabalho de Humanização. Disponível em:<http://www.hospitalsaopaulo.org.br/sites/humaniza/projeto_eq uilibrio_saude.php>. Acesso em: 12 abr. 2017.

HosPITAL SírIo-LIBANÊS. Cuidados Integrativos - Especialidades. Disponível em: <https://www.hospitalsiriolibanes.org.br/hospital/especialidades/nuc leo-cuidados-integrativos/Paginas/default.aspx>. Acesso em: 12 abr. 2017.

Hospital Sofia Feldman. NT Integrativas e Complementares. Disponível em: <http://www. sofiafeldman.org.br/atencao-a-mulher/ntintegrativas/>. Acesso em: 12 abr. 2017.

NúCLEO ANTHROPOS. Introdução à Medicina Integrativa. Disponível em: <http://www. nucleoanthropos.com/site/fundamentacaoteorica/medicina-integrativa.html>. Acesso em: 12 abr. 2017.

SINTE- SOCIEDADE INTERNACIONAL DE TERAPIAS. Dicionário de Técnicas. Disponível em: <http://www.sinte.com.br/publico-geral/dicionario-tecnicas>. Acesso em: 12 abr. 2017.

UNIFESP. NUMEPI. Disponível em:

<https://www.unifesp.br/reitoria/proex/acoes/nucleosassociados/numepi>. Acesso em: 12 abr. 2017.

UNIVERSIDADE ANHEMBI MORUMBI. Cursos - Naturologia. Disponível em: <http://portal.anhembi.br/cursos/naturologia/>. Acesso em: $17 \mathrm{abr}$. 2017.

UnIVERSIDADE do SUL DE SC. Cursos Presenciais e EaD - Naturologia. Disponível em:<http://www.unisul.br/wps/portal/home/ensino/graduacao/natu rologia/\#?unidade=11>. Acesso em: 17 abr. 2017.

TradTerm, São Paulo, v.37, n. 1, janeiro/2021, p. 265-293

Número Especial - Linguística de Corpus

www.revistas.usp.br/tradterm 
BRASIL: Ministério da Saúde. Portaria $n^{\circ}$ 971, de 03 de maio de 2006. Aprova a Política Nacional de Práticas Integrativas e Complementares (PNPIC) no Sistema Único de Saúde. Disponível em: <http://bvsms.saude.gov.br/bvs/saudelegis/gm/2006/prt0971_03_05 _2006.html>. Acesso em: 12 abr. 2017.

BrASIL: Ministério da Saúde. Política Nacional de Práticas Integrativas e Complementares no SUS. Brasília, DF: [s.n.], 2006. Disponível em: <http://bvsms.saude.gov.br/bvs/publicacoes/pnpic.pdf>. Acesso em: 12 abr. 2017.

\section{Referências do corpus em inglês}

BodDY, K. et al. Oxford Handbook of Complementary Medicine. Oxford University Press, 2008. 424 pgs.

SIXTH REPORT, 21 November 2000, By the Select Committee appointed to consider Science and Technology. Ordered to Report Complementary and Alternative Medicine. Disponível em: https://www.publications. parliament.uk/pa/ld199900/ldselect/ldscte ch/123/12301.htm Acesso em: 12 abr. 2017.

The General Regulatory Council for Complementary Therapies, The UK Federal Regulator for Complementary Therapies. Disponível em: http://www.grcct.org/Acesso em: 12 abr. 2017.

BRItish College of INTEgrative Medicine (BCIM). Disponível em: <http://www.integrativemedicine.uk.com/index.php>. Acesso em: 24 abr. 2017.

BRITISH SOCIETY OF INTEGRATED MEDICINE (BSIM). Disponível em: <http://www.bsim.org.uk/>. Acesso em: 24 abr. 2017.

CARDIFF METROPOLITAN UNIVERSITY. BSC (Hons) Complementary Healthcare Degree Course - Complementary Therapies. Disponível em: <http://www.cardiffmet.ac.uk/health/courses/Pages/Complementar y-Healthcare-BSc-(Hons).aspx>. Acesso em: 24 abr. 2017.

FEDERATION OF Holistic Therapists DiReCtORY SERVICE. Disponível em: <http://www.fht.org.uk/>. Acesso em: 24 abr. 2017.

Pure health School Of Natural Medicine International. Naturopath Natural Physician Diploma. Disponível em: $<$ https://purehealth.com/naturopathy-training-cambridgeengland/naturopath-natural-physician-diploma/>. Acesso em: 24 abr. 2017.

TradTerm, São Paulo, v.37, n. 1, janeiro/2021, p. 265-293

Número Especial - Linguística de Corpus www.revistas.usp.br/tradterm 
SCHOOL OF NATURAL MEDICINE Uk. Disponível em: <https://www.herenowhealing.com/>. Acesso em: 24 abr. 2017.

University College London Hospitals NHS Foundation TRUst (UCLH). Royal London Hospital for Integrated Medicine. Disponível em: <https://www.uclh.nhs.uk/OurServices/ServiceA-

Z/INTMED/Pages/Home.aspx>. Acesso em: 24 abr. 2017.

University OF GREenwich (Hadlow College). Equine Sports Therapy and Rehabilitation, BSC (Hons). Disponivel em: <http://www.gre.ac.uk/ug/pc/bsc-hons-equine-sports-therapy-andrehabilitation>. Acesso em: 24 abr. 2017.

Recebido em: 30/04/2020

Aceito em: 26/05/2020

Publicado em janeiro de 2021

TradTerm, São Paulo, v.37, n. 1, janeiro/2021, p. 265-293

Número Especial - Linguística de Corpus

www.revistas.usp.br/tradterm 